Wright State University

CORE Scholar

Mathematics and Statistics Faculty

Publications

Mathematics and Statistics

$12-2000$

\title{
Commuting Self-Adjoint Extensions of Symmetric Operators Defined from the Partial Derivatives
}

Palle Jorgensen

Steen Pedersen

Wright State University - Main Campus, steen.pedersen@wright.edu

Follow this and additional works at: https://corescholar.libraries.wright.edu/math

Part of the Applied Mathematics Commons, Applied Statistics Commons, and the Mathematics Commons

\section{Repository Citation}

Jorgensen, P., \& Pedersen, S. (2000). Commuting Self-Adjoint Extensions of Symmetric Operators Defined from the Partial Derivatives. Journal of Mathematical Physics, 41 (12), 8263-8278.

https://corescholar.libraries.wright.edu/math/32

This Article is brought to you for free and open access by the Mathematics and Statistics department at CORE Scholar. It has been accepted for inclusion in Mathematics and Statistics Faculty Publications by an authorized administrator of CORE Scholar. For more information, please contact library-corescholar@wright.edu. 


\title{
Commuting self-adjoint extensions of symmetric operators defined from the partial derivatives
}

\author{
Palle E. T. Jorgensen ${ }^{\text {a) }}$ \\ Department of Mathematics, The University of Iowa, Iowa City, Iowa 52242-1419 \\ Steen Pedersen ${ }^{\text {b) }}$ \\ Department of Mathematics, Wright State University, Dayton, Ohio 45435
}

(Received 24 May 2000; accepted for publication 5 September 2000)

\begin{abstract}
We consider the problem of finding commuting self-adjoint extensions of the partial derivatives $\left\{(1 / i)\left(\partial / \partial x_{j}\right): j=1, \ldots, d\right\}$ with domain $C_{c}^{\infty}(\Omega)$ where the selfadjointness is defined relative to $L^{2}(\Omega)$, and $\Omega$ is a given open subset of $\mathbb{R}^{d}$. The measure on $\Omega$ is Lebesgue measure on $\mathbb{R}^{d}$ restricted to $\Omega$. The problem originates with Segal and Fuglede, and is difficult in general. In this paper, we provide a representation-theoretic answer in the special case when $\Omega=I \times \Omega_{2}$ and $I$ is an open interval. We then apply the results to the case when $\Omega$ is a $d$ cube, $I^{d}$, and we describe possible subsets $\Lambda \subset \mathbb{R}^{d}$ such that $\left\{\left.e_{\lambda}\right|_{I^{d}}: \lambda \in \Lambda\right\}$ is an orthonormal basis in $L^{2}\left(I^{d}\right)$. (C) 2000 American Institute of Physics. [S0022-2488(00)02712-2]
\end{abstract}

\section{INTRODUCTION}

Recently several papers have appeared on commuting non-self-adjoint operators and their spectral theory; see, e.g., Ref. 1. The present paper concerns the case when the given commuting operators are unbounded and symmetric, but non-self-adjoint. A concrete class of operators is studied, and we address the questions of when commuting extension operators exist and, when they do exist, what their structural properties are.

The problem of understanding commuting symmetric, but non-self-adjoint, unbounded operators also has an origin in mathematical physics. ${ }^{2-5}$ The terminology from physics is "Hermitian," or "formally self-adjoint," for symmetry, i.e., for the identity $\langle S f \mid h\rangle=\langle f \mid S h\rangle$ for all vectors $f, h$ in the domain of the operator $S$. The simplest case of this is the problem of assigning quantum mechanical boundary conditions for free particles confined in a box. More specifically, the problem here corresponds to the quantum-mechanical trajectories of a particle confined to a region of tube type, e.g., a unit cube. It is "free" except for the boundary conditions, and variations of the boundary conditions (as considered here) correspond to different physics. For single operators, von Neumann solved (or made precise) the problem by use of the Cayley transform, and considering instead the extension problem for partial isometries. But this approach does not work well in the case of several operators. Powers (in Refs. 6 and 7) introduced an algebraic approach for understanding several operators, but the present problem is very concrete and does not lend itself easily to the algebraic techniques introduced by Powers.

Closely connected to the problem of finding commuting self-adjoint extensions of $(1 / i)\left(\partial / \partial x_{j}\right), j=1, \ldots, d$, on $C_{c}^{\infty}(\Omega)$ is the corresponding spectral question: If commuting selfadjoint extensions do exist, then it is known that the common eigenfunctions of the extension operators must be of the form $e_{\lambda}:=e^{i \lambda \cdot x}$ for special values of $\lambda \in \mathbb{R}^{d}$. Hence the spectral problem is that of finding when a given pair $(\Omega, \Lambda)$ satisfies the condition that $\left\{\left.e_{\lambda}\right|_{\Omega}: \lambda \in \Lambda\right\}$ is an orthogonal basis in the Hilbert space $\mathcal{L}^{2}(\Omega)$. We note that this so-called spectral pair condition is very restrictive, and so it explains the rigid geometric configurations $(\Omega, \Lambda)$ which admit solutions. But

\footnotetext{
${ }^{a)}$ Electronic mail: jorgen@math.uiowa.edu

b)Electronic mail: steen@math.wright.edu
} 
it also serves to motivate recent very interesting developments on overcomplete systems; see, e.g., Refs. 8 and 9.

The setting of spectral pairs in $d$ real dimensions involves two subsets $\Omega$ and $\Lambda$ in $\mathbb{R}^{d}$ such that $\Omega$ has finite and positive $d$-dimensional Lebesgue measure, and $\Lambda$ is an index set for an orthogonal $\mathcal{L}^{2}(\Omega)$-basis $e_{\lambda}$ of exponentials, i.e.,

$$
e_{\lambda}(x)=e^{i 2 \pi \lambda \cdot x}, \quad x \in \Omega, \quad \lambda \in \Lambda,
$$

where $\lambda \cdot x=\sum_{j=1}^{d} \lambda_{j} x_{j}$. We use vector notation $x=\left(x_{1}, \ldots, x_{d}\right), \lambda=\left(\lambda_{1}, \ldots, \lambda_{d}\right), x_{j}, \lambda_{j} \in \mathbb{R}, j$ $=1, \ldots, d$. The basis property refers to the Hilbert space $\mathcal{L}^{2}(\Omega)$ with inner product

$$
\langle f \mid g\rangle_{\Omega}:=\int_{\Omega} \overline{f(x)} g(x) d x,
$$

where $d x=d x_{1} \cdots d x_{d}$, and $f, g \in \mathcal{L}^{2}(\Omega)$. The corresponding norm is

$$
\|f\|_{\Omega}^{2}:=\langle f \mid f\rangle_{\Omega}=\int_{\Omega}|f(x)|^{2} d x,
$$

as usual. It follows that the spectral pair property for a pair $(\Omega, \Lambda)$ is equivalent to

$$
\Lambda-\Lambda=\left\{\lambda-\lambda^{\prime}: \lambda, \lambda^{\prime} \in \Lambda\right\}
$$

being contained in the zero-set of the complex function

$$
z \mapsto \int_{\Omega} e^{i 2 \pi z \cdot x} d x=: F_{\Omega}(z)
$$

where $z=\left(z_{1}, \ldots, z_{d}\right) \in \mathbb{C}^{d}$, and $z \cdot x:=\sum_{j=1}^{d} z_{j} x_{j}$, and the corresponding $e_{\lambda}$-set $\left\{e_{\lambda}: \lambda \in \Lambda\right\}$ being total in $\mathcal{L}^{2}(\Omega)$. Recall, totality means that the span of the $e_{\lambda}$ 's is dense in $\mathcal{L}^{2}(\Omega)$ relative to the $\|\cdot\|_{\Omega}$-norm, or, equivalently, that $f=0$ is the only $\mathcal{L}^{2}(\Omega)$-solution to

$$
\left\langle f \mid e_{\lambda}\right\rangle_{\Omega}=0, \text { for all } \lambda \in \Lambda \text {. }
$$

\section{SPECTRAL PAIRS}

The theory of spectral pairs was developed in previous joint papers by the coauthors. ${ }^{10-12} \mathrm{~A}$ set $\Omega$ with finite nonzero Lebesgue measure is called a spectral set if $(\Omega, \Lambda)$ is a spectral pair for some set $\Lambda$. We recall that Fuglede showed ${ }^{13}$ that the disk and the triangle in two dimensions are not spectral sets. By the disk and the triangle we mean the usual versions, respectively, $\left\{\left(x_{1}, x_{2}\right) \in \mathbb{R}^{2}: x_{1}^{2}+x_{2}^{2}<1\right\}$ and $\left\{\left(x_{1}, x_{2}\right) \in \mathbb{R}^{2}: 0<x_{1}, 0<x_{2}, x_{1}+x_{2}<1\right\}$. Note that, for the present discussion, it is inessential whether or not the sets $\Omega$ are taken to be open, but it is essential for the following theorem which we will need. It is due to Fuglede and the coauthors; see Refs. 10, $13-15$.

If $\Omega \subset \mathbb{R}^{d}$ is open, then we consider the partial derivatives $\partial / \partial x_{j}, j=1, \ldots, d$, defined on $C_{c}^{\infty}(\Omega)$ as unbounded skew-symmetric operators in $\mathcal{L}^{2}(\Omega)$. The corresponding versions $(1 / 2 \pi \sqrt{-1})\left(\partial / \partial x_{j}\right)$ are symmetric of course. We say that $\Omega$ has the extension property if there are commuting self-adjoint extension operators $H_{j}$, i.e.,

$$
\frac{1}{2 \pi i} \frac{\partial}{\partial x_{j}} \subset H_{j}, \quad j=1, \ldots, d .
$$

We say that the containment $A \subset B$ holds for two operators $A$ and $B$ if the graph of $A$ is contained in that of $B$. (For details, see Refs. 16 and 17.) Commutativity for the extension operators $H_{j}$ is in 
the strong sense of spectral resolutions. Since the $H_{j}$ 's are assumed self-adjoint, each one has a projection-valued spectral resolution $E_{j}$, i.e., an $\mathcal{L}^{2}(\Omega)$-projection-valued Borel measure on $\mathbb{R}$, such that $E_{j}(\mathrm{R})=I_{\mathcal{L}^{2}(\Omega)}$, and

$$
H_{j}=\int_{-\infty}^{\infty} \lambda E_{j}(d \lambda)
$$

for $j=1, \ldots, d$. The strong commutativity is taken to mean

$$
E_{j}(\Delta) E_{j^{\prime}}\left(\Delta^{\prime}\right)=E_{j^{\prime}}\left(\Delta^{\prime}\right) E_{j}(\Delta)
$$

for all $j, j^{\prime}=1, \ldots, d$, and all Borel subsets $\Delta, \Delta^{\prime} \subset \mathbb{R}$. Extensions commuting in a weaker sense were considered in Ref. 18.

Our analysis is based on von Neumann's deficiency-space characterization of the self-adjoint extensions of a given symmetric operator. ${ }^{19}$ Let $\Omega$ be an open set with finite Lebesgue measure. For each $j$, the deficiency spaces corresponding to $(1 / i)\left(\partial / \partial x_{j}\right)$ are infinite dimensional. It follows that each $(1 / i)\left(\partial / \partial x_{j}\right)$ has "many" self-adjoint extensions. The main problem (not addressed by von Neumann's theory) is the selection of a commuting set $H_{1}, H_{2}, \ldots, H_{d}$ of extensions. In fact, for some $\Omega$ (e.g., when $d=2$, the disk and the triangle) it is impossible to select a commuting set $H_{1}, H_{2}, \ldots, H_{d}$ of extensions.

We have (see Refs. 10, 13-15)

Theorem II.1 (Fuglede, Jorgensen, Pedersen): Let $\Omega \subset \mathbb{R}^{d}$ be open and connected with finite and positive Lebesgue measure. Then $\Omega$ has the extension property if and only if it is a spectral set. Moreover, with $\Omega$ given, there is a one-to-one correspondence between the two sets of subsets:

$$
\left\{\Lambda \subset \mathbb{R}^{d}:(\Omega, \Lambda) \text { is a spetral pair }\right\}
$$

and

$$
\begin{aligned}
& \qquad\left\{\Lambda \subset \mathbb{R}^{d}: \Lambda\right. \text { is the joint spectrum of some } \\
& \text { commutative family } \left.\left(H_{1}, \ldots, H_{d}\right) \text { of self-adjoint extensions }\right\} \text {. }
\end{aligned}
$$

This correspondence is determined as follows:

(a) If the extensions $\left(H_{1}, \ldots, H_{d}\right)$ are given, then $\lambda \in \Lambda$ if and only if

$$
e_{\lambda} \in \bigcap_{j} \text { domain }\left(H_{j}\right) .
$$

(b) If, conversely, $(\Omega, \Lambda)$ is a spectral pair at the outset, then the ansatz (2.6) and

$$
H_{j} e_{\lambda}=\lambda_{j} e_{\lambda}, \quad \lambda \in \Lambda
$$

determine uniquely a set of commuting extensions.

If $\Omega$ is only assumed open, then the spectral-set property implies the extension property, but not conversely.

Corollary II.2: Suppose $\Omega$ is open and connected. It follows then that a discrete set $\Lambda$ is the joint spectrum of some commuting self-adjoint extension operators $H_{j}, j=1, \ldots, d$, if and only if $(\Omega$,

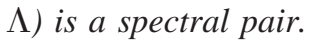

Remark II.3: The simplest case of a disconnected $\Omega$ which has the extension property, but which is not a spectral set, is in $d=1$, and we may take $\Omega=\langle 0,1\rangle \cup\langle 2,4\rangle$, i.e., the union of two intervals with a doubling and separation. The example was noted first in Ref. 13 and is based on the simple observation that the polynomial $1+z^{2}+z^{3}$ has no roots $\mathrm{z}$ on the circle $|z|=1$. 
Some of the interest in spectral pairs derives from their connection to tilings. A subset $\Omega \subset \mathbb{R}^{d}$ with nonzero measure is said to tile $\mathbb{R}^{d}$ if there is a set $L \subset \mathbb{R}^{d}$ such that the translates $\{\Omega+l: l$ $\in L\}$ cover $\mathbb{R}^{d}$ up to measure zero, and if the intersections

$$
(\Omega+l) \cap\left(\Omega+l^{\prime}\right) \text { for } l \neq l^{\prime} \text { in } L
$$

have measure zero. We will call $(\Omega, L)$ a tiling pair and we will say that $L$ is a set of translations. The spectral-set conjecture (see Refs. 13-15, 20, 21) states:

Conjecture II.4: Let $\Omega \subset \mathbb{R}^{d}$ have positive and finite Lebesgue measure. Then $\Omega$ is a spectral set if and only if there is a set $\mathrm{L}$ of translations which make $\Omega$ tile $\mathrm{R}^{d}$.

Lemma II.5: If $\Omega=I^{d}$, then the zero set for the function $F_{\Omega}$ in (1.4) is

$$
\mathbf{Z}_{I^{d}}=\left\{z \in \mathbb{C}^{d} \backslash\{0\}: \exists j \in\{1, \ldots, d\} \text { s.t. } z_{j} \in \mathbb{Z} \backslash\{0\}\right\} .
$$

Proof: The function $F_{I^{d}}(\cdot)$ factors as follows:

$$
F_{I^{d}}(z)=\prod_{j=1}^{d} e^{i \pi z_{j}} \frac{\sin \pi z_{j}}{\pi z_{j}}
$$

for $z=\left(z_{1}, \ldots, z_{d}\right) \in \mathbb{C}^{d}$, with the interpretation that the function $z \mapsto(\sin \pi z) / \pi z$ is 1 when $z=0$ in C.

Remark II.6: What is special about $\mathbf{Z}_{\Omega}$ for $\Omega=I^{d}$, as opposed to the general form of $\Omega$, is that $\mathbf{Z}_{I^{d}} \cup\{0\}$ is the Cayley graph of the group $\Gamma=Z^{d}$ with generators

$$
S=\{( \pm 1,0, \ldots, 0), \ldots,(0, \ldots, \pm 1,0, \ldots, 0), \ldots,(0, \ldots, 0, \pm 1)\} .
$$

We recall from Ref. 22, Chapter 10, the definition of the Cayley graph $G(\Gamma, S)$ of a discrete group $\Gamma$ with generators $\mathrm{S}, e \notin S$. When $\Gamma, \mathrm{S}$ are given, $G(\Gamma, S)$ is the graph with vertex set $\Gamma$ in which two vertices $\gamma_{1}, \gamma_{2}$ are the two ends of an edge iff $\gamma_{1}^{-1} \gamma_{2} \in S$. This gives a nonoriented graph, without any loop or multiple edge.

\section{TWO DIMENSIONS}

We begin with the following simple observation in one dimension for $\Omega=I=[0,1\rangle$. (For details, see Refs. 10 and 16.)

Proposition III.1: The only subsets $\Lambda \subset \mathbb{R}$ such that $(I, \Lambda)$ is a spectral pair are the translates

$$
\Lambda_{\alpha}:=\alpha+\mathbb{Z}=\{\alpha+n: n \in \mathbb{Z}\},
$$

where $\alpha$ is some fixed real number.

In two dimensions, the corresponding result is more subtle, but the possibilities may still be enumerated as follows:

Theorem III.2 (Ref. 23): The only subsets $\Lambda \subset \mathbb{R}^{2}$ such that $\left(I^{2}, \Lambda\right)$ is a spectral pair must belong to either one or the other of the two classes, indexed by a number $\alpha$, and a sequence $\left\{\beta_{m} \in[0,1\rangle: m \in \mathbb{Z}\right\}$, where

$$
\Lambda=\left\{\left(\begin{array}{c}
\alpha+m \\
\beta_{m}+n
\end{array}\right): m, n \in \mathbb{Z}\right\}
$$

or

$$
\Lambda=\left\{\left(\begin{array}{c}
\beta_{n}+m \\
\alpha+n
\end{array}\right): m, n \in \mathbb{Z}\right\} .
$$

Each of the two types occurs as the spectrum of a pair for the cube $I^{2}$, and each of the sets $\Lambda$ as specified is a set of translation vectors which produces a tiling of $\mathbb{R}^{2}$ by the cube $I^{2}$. 


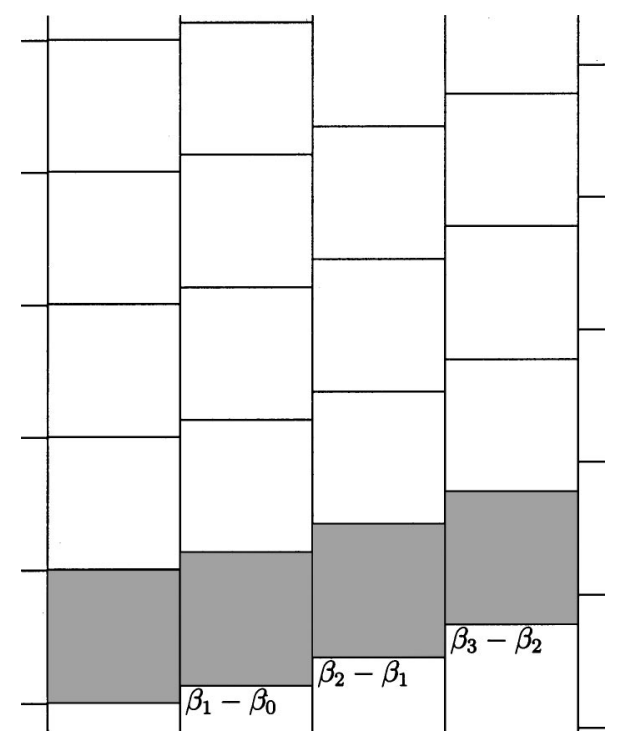

FIG. 1. Illustrating tiling with (3.2).

Proof: See Ref. 23 for details. The following are some remarks of relevance to the general extension problem for operators.

The assertion in the theorem about $\Lambda$ translations tiling the plane with $I^{2}$ is also clear from (3.2) and (3.3), and is illustrated graphically in Figs. 1 and 2.

It is clear that the pattern (3.2) for $d=2$ continues to higher dimensions as follows:

$$
\left(\begin{array}{c}
\alpha+k_{1} \\
\beta\left(k_{1}\right)+k_{2} \\
\gamma\left(k_{1}, k_{2}\right)+k_{3} \\
\vdots \\
\zeta\left(k_{1}, k_{2}, \ldots, k_{d-1}\right)+k_{d}
\end{array}\right)
$$

with $k_{1}, k_{2}, \ldots, k_{d} \in \mathbb{Z}$, and

$$
\begin{gathered}
\beta: \mathbb{Z} \rightarrow[0,1\rangle, \\
\gamma: \mathbb{Z} \times \mathbb{Z} \rightarrow[0,1\rangle, \\
\vdots \\
\zeta: Z^{d-1} \rightarrow[0,1\rangle .
\end{gathered}
$$

Of course, then there are the obvious modifications of those cases resulting from permutation of the $d$ coordinates; but the assertion is that, when $d \geqslant 10$, these configurations do not suffice for cataloguing all the possible spectra $\Lambda$ which turn $\left(I^{d}, \Lambda\right)$ into an $\mathbb{R}^{d}$-spectral pair.

We now turn to the nontrivial spectral-theoretic content of the conclusion of the theorem. We claim that the two cases (3.2) and (3.3) suffice when $d=2$. Note that the sequence $\beta: Z \rightarrow[0,1\rangle$ is completely arbitrary.

We will show in Theorem V.1 that, up to a single translation in the plane, the possibilities for the coordinates of points in a spectrum $\Lambda$ for $I^{2}$ are given by two sequences $\xi_{m}, \eta_{n}$ satisfying the following two cocycle relations:

$$
\left(e^{i \xi_{m+k}-e^{i \xi_{m}}}\right)\left(1-e^{i \eta_{n}}\right)=0
$$




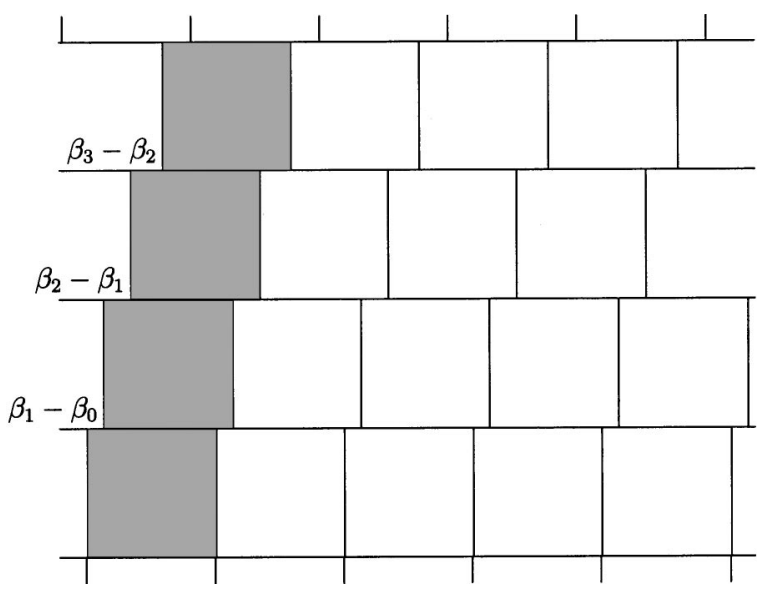

FIG. 2. Illustrating tiling with (3.3).

and

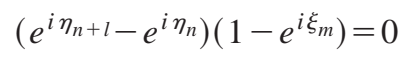

as identities in $m, n \in \mathbb{Z}$, and $k, l \in \mathbb{Z}\{\{0\}$. Note that the respective sequences are determined from this only up to $2 \pi \mathbb{Z}$ at each coordinate place.

Simple algebra shows that the two identities (3.5) and (3.6) imply the following single identity:

$$
\left(1-e^{i \xi_{m}+k}\right)\left(1-e^{i \eta_{n}}\right)=\left(1-e^{i \xi_{m}}\right)\left(1-e^{i \eta_{n+l}}\right)
$$

again for all $m, n \in \mathbb{Z}$ and $k, l \in \mathbb{Z} \backslash\{0\}$. But it follows from (3.7) that at least one of the two sequences, $1-e^{i \xi_{m}}$ or $1-e^{i \eta_{n}}$, must then vanish identically. This yields the connection to the two cases for $\Lambda$ stated in (3.2) and (3.3) of the theorem.

Hence the result giving two classes for $\Lambda$ in Theorem III.2 may be derived from our more general result in Sec. V. V.

The proof sketch of Theorem III.2 is completed for now, but details will be resumed in Sec.

\section{OPERATOR EXTENSIONS}

We saw in Theorem II.1 that in some cases the existence problem for spectral pairs, i.e., the question of when some given open subset $\Omega$ in $\mathbb{R}^{d}$ has an orthogonal basis $\left\{e_{\lambda}: \lambda \in \Lambda\right\}$ in $\mathcal{L}^{2}(\Omega)$ for some set $\Lambda$ in $\mathbb{R}^{d}$, may be reformulated as a problem about existence of commuting selfadjoint extensions of the operators $\left\{(1 / i)\left(\partial / \partial x_{j}\right): j=1, \ldots, d\right\}$ with common (dense) domain $C_{c}^{\infty}(\Omega)$ in $\mathcal{L}^{2}(\Omega)$. Suppose for the moment that $\Omega=\langle 0,1\rangle \times \Omega_{2}$ where $\Omega_{2}$ is some subset in $\mathbb{R}^{d-1}$ of finite positive $(d-1)$-dimensional Lebesgue measure. We then have the following classification of the self-adjoint extensions $H$ of $(1 / i)\left(\partial / \partial x_{1}\right)$.

Theorem IV.1: The symmetric operator $(1 / i)\left(\partial / \partial x_{1}\right)$ in $\mathcal{L}^{2}\left(\langle 0,1\rangle \times \Omega_{2}\right)$ with dense domain $\mathcal{D}$ consisting of $\varphi \in \mathcal{L}^{2}\left(\langle 0,1\rangle \times \Omega_{2}\right)$ such that $\varphi(\cdot, y) \in C_{c}^{\infty}(\langle 0,1\rangle)$ for all $y \in \Omega_{2}$, has self-adjoint extensions indexed by unitary operators $V$ in $\mathcal{L}^{2}\left(\Omega_{2}\right)$ in such a way that the (unique) extension $H_{V}$ is determined by its core domain being of the form

$$
\mathcal{D}_{V}=\left\{\varphi\left(x_{1}, y\right)+e^{x_{1}} h(y)+e^{1-x_{1}}(V h)(y): \varphi \in \mathcal{D}, h \in \mathcal{L}^{2}\left(\Omega_{2}\right)\right\}
$$

and 


$$
i H_{V}\left(\varphi+e^{x_{1}} h+e^{1-x_{1}} V h\right)=\frac{\partial \varphi}{\partial x_{1}}+e^{x_{1}} h-e^{1-x_{1}} V h,
$$

for $\varphi \in \mathcal{D}$ and $h \in \mathcal{L}^{2}\left(\Omega_{2}\right)$. We shall interpret the implicit boundary condition dictating some extension $H_{V}$ as

$$
f(1, \cdot)=U_{V}(f(0, \cdot))
$$

$f \in \mathcal{D}_{V}$ where the partial isometry $U_{V}$ is given by

$$
W_{V}=(e I+V)(I+e V)^{-1}, \quad U_{V}=\exp W_{V} .
$$

Conversely, $V$ may be calculated from $U_{V}$ by

$$
V=\left(I-e W_{V}\right)^{-1}\left(W_{V}-e I\right)
$$

and in each case, the fractional linear transform, and its inverse, are well defined.

Proof: The proof is based on von Neumann's deficiency-space analysis of self-adjoint extensions, and we refer to Refs. 16, 19, and 24 for background material on the theory of operator extensions. If $S$ is a symmetric operator with dense domain $\mathcal{D}$ in a Hilbert space $\mathcal{H}$, then it has self-adjoint extension if and only if the two spaces

$$
((i I \pm S) \mathcal{D})^{\perp}=: \mathcal{D}_{ \pm}
$$

have the same dimensions. In that case, the corresponding extensions are given by partial isometries between the respective defect spaces $\mathcal{D}_{+}$and $\mathcal{D}_{-}$(see Ref. 16, 17, or 19). For convenience, we have chosen a slightly different "normalization" in our treatment of the Cayley transform (4.4) and its inverse (4.5). We did not normalize the functions $e^{x_{1}}$ and $e^{1-x_{1}}$ in the defect spaces. They have $\mathcal{L}^{2}(I)$-norm equal to

$$
\left(\frac{e^{2}-1}{2}\right)^{1 / 2}
$$

The fact that $U_{V}$ in (4.4) then defines a partial isometry as claimed amounts to the identities:

If $\psi\left(x_{1}, y\right)=e^{x_{1}} h(y)+e^{1-x_{1}}(V h)(y)$ as in (4.1), then

$$
\psi(1, y)=e h(y)+(V h)(y)=(e I+V) h,
$$

and

$$
\psi(0, y)=h(y)+e V h(y)=(I+e V) h
$$

This means that the vectors in the domain (4.1) are given by the boundary conditions (4.3) which in turn determine the unitary one-parameter group

$$
\mathcal{U}_{V}(t):=\exp \left(i t H_{V}\right), \quad t \in \mathbb{R} .
$$

This group is defined from (4.3) by using translation modulo $\mathbb{Z}$ in the $x_{1}$ variable. Then the operator $U_{V}$ in (4.4) is used in defining the representation $\mathbb{R} \ni t \mapsto \mathcal{U}_{V}(t)$ via induction from $\mathbb{Z}$.

If $V: \mathcal{D}_{+} \rightarrow \mathcal{D}_{-}$is a partial isometry, then the domain of the corresponding extension $H(H$ $\left.=H_{V}\right)$ is

$$
\left\{\varphi+h_{+}+V h_{+}: \varphi \in \mathcal{D}, h_{+} \in \mathcal{D}_{+}\right\}
$$

and 


$$
i H_{V}\left(\varphi+h_{+}+V h_{+}\right)=i S \varphi+h_{+}-V h_{+}
$$

It follows that the lemma amounts to an identification of the defect spaces $\mathcal{D}_{ \pm}$when the symmetric operator is as specified. When the variables in $\Omega=\langle 0,1\rangle \times \Omega_{2}$ are separated as $\left(x_{1}, y\right)$, $0<x_{1}<1, y=\left(x_{2}, \ldots, x_{d}\right) \in \Omega_{2}$, then vectors $h_{ \pm} \in \mathcal{D}_{ \pm}$are precisely the solutions to

$$
S^{*} h_{ \pm}= \pm i h_{ \pm}
$$

This amounts to solving

$$
\frac{\partial}{\partial x_{1}} h_{ \pm}\left(x_{1}, y\right)= \pm h_{ \pm}\left(x_{1}, y\right)
$$

in the sense of distributions, but with the restrictions $h_{ \pm} \in \mathcal{L}^{2}\left(\langle 0,1\rangle \times \Omega_{2}\right)$. The result of the lemma then follows from von Neumann's characterization. If the minimal operator is not closed at the outset, then the resulting self-adjoint extension comes from passing to the operator closure in the formulas (4.2) and (4.7).

Corollary IV.2: Let $V$ be a unitary operator in $\mathcal{L}^{2}\left(\Omega_{2}\right)$ and let $H_{V}$ be the self-adjoint extension operator described in Theorem IV.1 in (4.2) and (4.3). Then $H_{V}$ generates a unitary oneparameter group $\left\{U_{V}(t): t \in \mathbb{R}\right\}$ in $\mathcal{L}^{2}\left(\langle 0,1\rangle \times \Omega_{2}\right)$ which may be realized (up to unitary equivalence) in the Hilbert space $\mathcal{H}_{V}$ of measurable functions $f: \mathbb{R} \rightarrow \mathcal{L}^{2}\left(\Omega_{2}\right)$, satisfying

$$
f\left(x_{1}+1\right)=U_{V}\left(f\left(x_{1}\right)\right),
$$

for all $x_{1} \in \mathbb{R}$, where $U_{V}$ is the operator from (4.4) in Theorem IV.1, and the norm on $\mathcal{H}_{V}$ is defined by

$$
\|f\|_{\mathcal{H}_{V}}^{2}=\int_{0}^{1}\left\|f\left(x_{1}\right)\right\|_{\mathcal{L}^{2}\left(\Omega_{2}\right)}^{2} d x_{1}
$$

In this space the group $U_{V}(t): \mathcal{H}_{V} \rightarrow \mathcal{H}_{V}$ is given by

$$
\left(U_{V}(t) f\right)\left(x_{1}\right)=f\left(x_{1}-t\right) \text { for } x_{1}, t \in \mathbb{R} \text {. }
$$

The unitary isomorphism of $\mathcal{H}_{V}$ onto $\mathcal{L}^{2}\left(\langle 0,1\rangle \times \Omega_{2}\right)=\mathcal{L}^{2}\left(\langle 0,1\rangle, \mathcal{L}^{2}\left(\Omega_{2}\right)\right)$ is simply the restriction to $\langle 0,1\rangle$ in the $x_{1}$-variable. Finally, if $U_{V}(t)$ is computed in $\mathcal{L}^{2}\left(\langle 0,1\rangle \times \Omega_{2}\right)$, the formula is

$$
\left(U_{V}(t) f\right) f\left(x_{1}, \cdot\right)=\left\{\begin{array}{l}
f\left(x_{1}-t, \cdot\right) \quad \text { if } 0 \leqslant t<x_{1}<1 \\
U_{V}\left(f\left(x_{1}-t, \cdot\right)\right) \quad \text { if } 0<x_{1} \leqslant t \leqslant 1
\end{array} .\right.
$$

Proof: The realization on the space $\mathcal{H}_{V}$ is the interpretation of $U_{V}$ as a unitary representation of the group $\mathrm{R}$ which is induced from the subgroup $\mathbb{Z}$ via formula (4.10). The advantage of this viewpoint is that the spectral resolution of the unitary operator $U_{V}$ leads directly to an associated direct integral decomposition for the unitary one-parameter group $\left\{U_{V}(t): t \in \mathbb{R}\right\}$ which is generated by the extension operator $H_{V}$.

When the corollary is applied to $\mathcal{L}^{2}(I \times I)$ from Sec. III we note that the respective unitary one-parameter groups, $U_{x}(s)$ and $U_{y}(t)$, on $\mathcal{L}^{2}\left(I^{2}\right)$ which are generated by self-adjoint extension operators of $(1 / i)(\partial / \partial x)$ and $(1 / i)(\partial / \partial y)$ with domain $C_{c}^{\infty}\left(I^{2}\right)$, are induced representations in the sense of (4.9) and (4.10). For the extensions of $(1 / i)(\partial / \partial x)$, the boundary-unitary from (4.9) is acting on $\mathcal{L}^{2}(\{0<y<1\})$. But we shall view it as a unitary operator in $\mathcal{L}^{2}(I \times I)=$ $\mathcal{L}^{2}\left(I_{x}\right) \otimes \mathcal{L}^{2}\left(I_{y}\right)$ via $U \leftrightarrow I \otimes U_{2}$ with $U_{2}$ acting in the $y$ variable. A similar observation applies to the unitary one-parameter group $\left\{U_{y}(t): t \in \mathbb{R}\right\}$ acting on $\mathcal{L}^{2}\left(I^{2}\right)$ and generated by one of the self-adjoint extensions of $(1 / i)(\partial / \partial y)$. Hence the boundary conditions for $\left\{U_{x}(s): s \in \mathbb{R}\right\}$ are given 
by a unitary $U \simeq I \otimes U_{2}$ with $U_{2}$ acting in the second variable, while those of $\left\{U_{y}(t): t \in \mathbb{R}\right\}$ are determined by a second unitary operator $V$ in $\mathcal{L}^{2}\left(I^{2}\right)$, now of the form $V \leftrightarrow V_{1} \otimes I$ with $V_{1}$ acting in the first variable of $\mathcal{L}^{2}(I \times I)$.

With this terminology we have the following preliminary result for the square $I^{2}$ in the plane.

Theorem IV.3: Let $U_{x}(s)$ be the unitary one-parameter group on $\mathcal{L}^{2}(I \times I)$, and let $U_{2}$ be the corresponding unitary boundary operator acting in the second variable $y$. Then $U_{2}$ commutes with the phase-periodic translation in the $y$ variable for a phase angle $\beta$ if and only if there is a real-valued sequence $\left\{\varphi_{n}: n \in \mathbb{Z}\right\}$ such that

$$
U_{x}(s) e_{m+\varphi_{n}} \otimes e_{n+\beta}=e^{i 2 \pi\left(m+\varphi_{n}\right) s} e_{m+\varphi_{n}} \otimes e_{n+\beta}
$$

for all $s \in \mathbb{R}$ and $m, n \in \mathbb{Z}$, where for $(\xi, \eta) \in \mathbb{R}^{2}, e_{\xi} \otimes e_{\eta}(x, y)=e_{\xi}(x) e_{\eta}(y)=e^{i 2 \pi(\xi x+\eta y)}$, restricted to $(x, y) \in I^{2}$.

Proof: Recall that some fixed unitary one-parameter group $\left\{U_{x}(s): s \in \mathbb{R}\right\}$ on $\mathcal{L}^{2}(I \times I)$ is determined uniquely by the corresponding boundary operator $I \otimes U_{2}$. But it follows from Proposition III.1 that $U_{2}$ satisfies the commutativity property of the theorem if and only if it is diagonalized by the basis functions $\left\{e_{n+\beta}: n \in \mathbb{Z}\right\}$ in $\mathcal{L}^{2}\left(I_{y}\right)$ for some $\beta \in[0,1\rangle$, i.e., if, for some sequence $\varphi_{n}$,

$$
\left(U_{2} e_{n+\beta}\right)(y)=e^{i 2 \pi \varphi_{n}} e_{n+\beta}(y) .
$$

But, according to Corollary IV.2, this means that $U_{x}(s)$ as an induced representation decomposes accordingly, which is to say that the basis vectors $e_{m+\varphi_{n}} \otimes e_{n+\beta}$ simultaneously diagonalize each operator $U_{x}(s)$ as stated in formula (4.12).

Remark IV.4: For more details on the operator-theoretic approach to spectrum and to tiles, we refer to Refs. 15 and $25-27$.

\section{COCYCLES IN TWO DIMENSIONS}

In this section, we continue with the self-adjoint extensions of the two commuting minimal operators $(1 / i)(\partial / \partial x)$ and $(1 / i)(\partial / \partial y)$ with common dense domain $C_{c}^{\infty}\left(I^{2}\right)$ in $\mathcal{L}^{2}\left(I^{2}\right)$.

Theorem V.1: Consider two commuting unitary one-parameter groups $U_{x}(s)$ and $U_{y}(t)$ on $\mathcal{L}^{2}(I \times I)$ with respective boundary unitaries $U_{2}$ and $V_{1}$. Then:

(i) Either $U_{2}$ is of the form $a I_{\mathcal{L}^{2}\left(I_{y}\right)}$ for a scalar $a$, or else $V_{1}$ commutes with periodic translation in the $x$-variable.

(ii) Either $V_{1}$ is of the form $b I_{\mathcal{L}^{2}\left(I_{x}\right)}$ for some scalar $b$, or else $U_{2}$ commutes with periodic translation in the y-variable.

(iii) In case $U_{2}=e^{i 2 \pi \alpha} I_{\mathcal{L}^{2}\left(I_{y}\right)}$, then

$$
U_{x}(s)\left(e_{\alpha+m} \otimes g\right)=e^{i 2 \pi(\alpha+m) s} e_{\alpha+m} \otimes g
$$

for all $m \in \mathbb{Z}$ and $g \in \mathcal{L}^{2}\left(I_{y}\right)$.

(iv) In case $V_{1}=e^{i 2 \pi \beta} I_{\mathcal{L}^{2}\left(I_{x}\right)}$, then

$$
U_{y}(t)\left(f \otimes e_{\beta+n}\right)=e^{i 2 \pi(\beta+n) t} f \otimes e_{\beta+n}
$$

for all $f \in \mathcal{L}^{2}\left(I_{x}\right)$ and $n \in \mathbb{Z}$.

Remark V.2: It follows that the conclusion in Theorem IV.3 is satisfied when the two oneparameter groups commute, i.e., when

$$
U_{x}(s) U_{y}(t)=U_{y}(t) U_{x}(s)
$$

is assumed, $s, t \in \mathbb{R}$. Specifically, it will then always be the case that $U_{2}$ commutes with some phase-periodic translation in the y-variable, while $V_{1}$ commutes with some (possibly different) 
phase-periodic translation in the $\mathrm{x}$-variable. [Also note that (5.3) is a reformulation of (2.3) in the case $d=2$. Furthermore (5.3) signifies the presence of a unitary representation of $\mathbb{R}^{2}$.]

Proof of Theorem V.1: When the two one-parameter groups $U_{x}(s)$ and $U_{y}(t)$ are written in the form (4.11) from Corollary IV.2, then the alternatives in (4.11) may be expanded as follows. Let $\tau_{s}$ denote periodic translation in $\mathcal{L}^{2}(\langle 0,1\rangle)$, and let $P_{s}$ denote the projection of $\mathcal{L}^{2}(\langle 0,1\rangle)$ onto $\mathcal{L}^{2}(\langle 0, s\rangle)$, with $P_{s}^{\perp}=I-P_{s}$ denoting then the projection onto the complement $\mathcal{L}^{2}(\langle s, 1\rangle)$, for $s$ $\in[0,1]$. We have $P_{0}=0$ and $P_{1}=I_{\mathcal{L}^{2}(\langle 0,1\rangle)}$. Then from (4.11) we get

$$
U_{x}(s)=\tau_{s} P_{s}^{\perp} \otimes I+\tau_{s} P_{s} \otimes U_{2}
$$

and

$$
U_{y}(t)=I \otimes \tau_{t} P_{t}^{\perp}+V_{1} \otimes \tau_{t} P_{t} .
$$

The assumed commutativity (5.3) then takes the form:

$$
\begin{aligned}
& \tau_{s} P_{s}^{\perp} V_{1} \otimes \tau_{t} P_{t}+\tau_{s} P_{s} \otimes U_{2} \tau_{t} P_{t}^{\perp}+\tau_{s} P_{s} V_{1} \otimes U_{2} \tau_{t} P_{t} \\
& \quad=\tau_{s} P_{s} \otimes \tau_{t} P_{t}^{\perp} U_{2}+V_{1} \tau_{s} P_{s}^{\perp} \otimes \tau_{t} P_{t}+V_{1} \tau_{s} P_{s} \otimes \tau_{t} P_{t} U_{2} .
\end{aligned}
$$

If $V_{1}$ is not a scalar times $I_{\mathcal{L}^{2}\left(I_{x}\right)}$ then two terms on either side are independent when evaluated on $f \otimes g$. Hence both $U_{2} \tau_{t} P_{t}^{\perp}=\tau_{t} P_{t}^{\perp} U_{2}$ and $U_{2} \tau_{t} P_{t}=\tau_{t} P_{t} U_{2}$ hold. Addition of these two identities yields $U_{2} \tau_{t}=\tau_{t} U_{2}$ which is the commutativity of $U_{2}$ with periodic translation.

If on the other hand $V_{1}$ is a scalar, then it follows from the argument in Sec. IV that (iv) must hold.

The two possibilities for the other boundary operator $U_{2}$ lead to cases (i) and (iii) by symmetry.

Corollary V.3: Consider unitary one-parameter groups $U_{x}(s)$ and $U_{y}(t)$ as in Theorem V.I and suppose the corresponding boundary operators $U_{2}$ and $V_{1}$ diagonalize as follows (identities in $n, m \in \mathbb{Z})$ :

$$
U_{2} e_{n+\beta}=e^{i 2 \pi \alpha_{n}} e_{n+\beta}
$$

and

$$
V_{1} e_{m+\alpha}=e^{i 2 \pi \beta_{m}} e_{m+\alpha}
$$

for some $\alpha, \beta \in \mathbb{R}$. The sequences $\alpha_{n}, \beta_{m}$ will be chosen taking values in $[0,1\rangle$. Then the commutativity (5.3) for the two groups holds if and only if the two sequences satisfy a certain cocycle property: Let $a_{n}:=e^{i 2 \pi \alpha_{n}}$ and $b_{m}:=e^{i 2 \pi \beta_{m}}$. Then the two identities

$$
\left(b_{m}-b_{m+k}\right)\left(1-a_{n}\right)=0, \quad m, n \in \mathbb{Z}, \quad k \in \mathbb{Z} \backslash\{0\}
$$

and

$$
\left(a_{n}-a_{n+l}\right)\left(1-b_{m}\right)=0, \quad m, n \in \mathbb{Z}, \quad l \in \mathbb{Z} \backslash\{0\}
$$

are equivalent to the commutativity (5.3). If commutativity holds, we must have $\left(1-a_{n}\right)$. $\left(1-b_{m}\right) \equiv 0, n, m \in \mathbb{Z}$. Hence we get a spectral pair with spectrum $\Lambda$ having one of the two forms

$$
\left\{\left(\begin{array}{c}
\alpha+m \\
n+\beta_{m}
\end{array}\right): m, n \in \mathbb{Z}\right\} \quad \text { if } \alpha_{n} \equiv 0,
$$




$$
\left\{\left(\begin{array}{c}
m+\alpha_{n} \\
\beta+n
\end{array}\right): m, n \in \mathbb{Z}\right\} \quad \text { if } \beta_{m} \equiv 0
$$

The derivation of the two cocycle identities (5.8) and (5.9) from commutativity (5.3) at the end of the proof is based on the following corollary of independent interest:

Corollary V.4: Let $U=1 \otimes U_{2}$ and $V=V_{1} \otimes I$ be the respective boundary operators of the one-parameter unitary groups $U_{x}(s)$ and $U_{y}(t)$ acting on $\mathcal{L}^{2}(I \times I)$. Then, if (5.6) and (5.7) hold for some $\alpha, \beta$ and some sequences as specified, it follows that the respective one-parameter groups may be expanded in the common basis $E(m, n)=E_{(\alpha, \beta)}(m, n):=e_{m+\alpha}^{(1)} \otimes e_{n+\beta}^{(2)},(m, n)$ $\in Z^{2}$, as follows: There are complex sequences $\left\{s_{k}\right\}_{k \in \mathbb{Z}}$ and $\left\{t_{l}\right\}_{l \in \mathbb{Z}}$ so that, if we define

$$
s_{0}^{\perp}:=1-s_{0}, \quad t_{0}^{\perp}:=1-t_{0}
$$

and

$$
s_{k}^{\perp}:=-s_{k}(\text { for } k \neq 0), \quad t_{l}^{\perp}:=-t_{l} \quad(\text { for } l \neq 0),
$$

then

$$
U_{x}(s) E(m, n)=\sum_{k \in \mathbb{Z}} e^{i 2 \pi(m+\alpha+k) s}\left(s_{k}^{\perp}+s_{k} a_{n}\right) E(m+k, n)
$$

and

$$
U_{y}(t) E(m, n)=\sum_{l \in Z} e^{i 2 \pi(n+\beta+l) t}\left(t_{l}^{\perp}+t_{l} b_{m}\right) E(m, n+l) .
$$

The two one-parameter groups $U_{x}(s)$ and $U_{y}(t)$ commute if and only if the cocycle identities (5.8) and (5.9) hold.

Proof: Recall from (5.4) and (5.5) that the two one-parameter groups are expressed in terms of multiplication operators on $\mathcal{L}^{2}(\langle 0,1\rangle)$ with the respective indicator functions $\chi_{\langle 0, s\rangle}$ and $\chi_{\langle 0, t\rangle}$. The sequences (5.10)-(5.11) are the Fourier coefficients of these indicator functions, acting by multiplication in $\mathcal{L}^{2}(I)$, and the relations (5.10) and (5.11) simply reflect the following two obvious identities:

$$
\chi_{\langle 0, s\rangle}+\chi_{[s, 1\rangle}=1
$$

and

$$
\chi_{\langle 0, t\rangle}+\chi_{[t, 1\rangle}=1,
$$

as functions on the unit interval. When the resulting formulas (5.12) and (5.13) are substituted into

$$
U_{x}(s) U_{y}(t) E(m, n)=U_{y}(t) U_{x}(s) E(m, n)
$$

the equivalence to (5.8) and (5.9) results.

\section{QUASICRYSTALS}

For the spectral pairs $\left(I^{d}, \Lambda\right)$ in dimensions $d=2,3$, we noted that each of the candidates for spectrum $\Lambda$ tiles $\mathbb{R}^{d}$ with $\Lambda$-translates of $I^{d}$. (See Theorems III.2 and IV.3.) But reviewing formulas (3.2)-(3.3) and (3.4), and (7.4) in the next section, for the possible sets $\Lambda$ which serve as $I^{d}$ spectrum, we find functions $\alpha, \beta, \ldots$ on $\mathbb{Z}$ or $\mathbb{Z}^{k}$ which describe the particular set $\Lambda$. Since all the candidates for $\Lambda$ make tailings, there is a direct geometric interpretation for these functions; but we note in the present section that there is also a spectral-theoretic significance which derives from diffraction considerations of quasicrystals; see Refs. 28-30. 
In this setting, diffractions show up as discrete components of the spectral distribution

$$
D_{\Lambda}(x)=\sum_{\lambda \in \Lambda} e_{\lambda}(x)=\sum_{\lambda \in \Lambda} e^{i 2 \pi \lambda \cdot x} .
$$

We say that a spectrum $\Lambda\left(\subset \mathbb{R}^{d}\right)$ has a diffraction pattern if there is a pair $(M, c)$ where $M$ is a subset of $\mathbb{R}^{d}$ and $c$ is a function (measuring intensity) defined on $M$ such that

$$
D_{\Lambda}(x)=\sum_{m \in M} c(\mu) \delta(x-\mu)
$$

i.e., the spectral distribution is a weighted sum of point masses, supported on some (discrete) subset $M$ in $\mathbb{R}^{d}$. Note that the interpretation in both of the summations involving $D_{\Lambda}(\cdot)$ is to be understood as Schwartz distributions; that is if the respective sums are evaluated on a testing function $\varphi \in C_{c}^{\infty}\left(\mathbb{R}^{d}\right)$, then the first sum yields $\Sigma_{\lambda \in \Lambda} \widetilde{\varphi}(\lambda)$ where $\widetilde{\varphi}(\lambda)=\int_{\mathbb{R}^{d}} e_{\lambda}(x) \varphi(x) d x$, while the second sum is $\sum_{\mu \in M} c(\mu) \varphi(\mu)$. We also note that, by the Poisson summation formula, the condition is satisfied if $\Lambda=\mathbb{Z}^{d}=M$, and the density (intensity) function $c$ is $c \equiv 1$ on $M$.

We shall also need the following definition: A function $\xi$ on $\mathbb{R}$ is said to be quasiperiodic if there are positive numbers $\omega_{1}, \ldots, \omega_{r}$, which are independent over $\mathbb{Q}$, and functions $\xi_{1}, \ldots, \xi_{r}$ such that $\xi_{j}$ has $\omega_{j}$ as period, and $\xi=\sum_{j=1}^{r} \xi_{j}$. The condition on $\xi_{j}$ amounts to the generalized Fourier expansion

$$
\xi_{j}(x)=\sum_{n \in \mathbb{Z}} c_{j}(n) \exp \left(i 2 \pi \frac{n x}{\omega_{j}}\right)
$$

In the following result we show that, if the functions which define a spectrum $\Lambda$ for some $I^{d}$ are quasiperiodic, then it follows that $\Lambda$ has a diffraction pattern. We will not state the result in the widest generality as it will be clear that the idea in the simplest case carries over to the variations in higher dimensions. Even for $d=2$, Theorem III.2 shows that there are two classes of $\Lambda$ corresponding to (3.2) and (3.3), respectively. In the following we will treat only (3.2), but the result applies to (3.3) mutatis mutandis.

Theorem VI.1: Let

$$
\Lambda=\left\{\left(\begin{array}{c}
m \\
\beta(m)+n
\end{array}\right): m, n \in \mathbb{Z}\right\}
$$

for some function $\beta: \mathbb{Z} \rightarrow \mathbb{R}$ and suppose $\beta$ extends to a function on $\mathbb{R}$ which is quasiperiodic with periods $\omega_{1}, \ldots, \omega_{r}$, independent over $\mathrm{Q}$. Then it follows that $\left(I^{2}, \Lambda\right)$ is a spectral pair with diffraction pattern, specifically, there is a density function $c: \mathbb{Z}^{r} \times \mathbb{Z} \rightarrow \mathrm{C}$ such that

$$
D_{\Lambda}(x, y)=\sum_{k \in \mathbb{Z}^{r}} \sum_{n \in \mathbb{Z}} c(k, n) \sum_{n \in \mathbb{Z}} \delta\left(x-\sum_{i=1}^{r} \frac{k_{i}}{\omega_{i}}-m\right) \otimes \delta(y-n)
$$

with the density $c\left(k_{1}, \ldots, k_{r}, n\right)$ derived from the Bohr almost periodic Fourier expansion applied to $\beta$.

Proof: Consider the formula $D_{\Lambda}(x, y)=\Sigma_{m} \Sigma_{n} e^{i 2 \pi(m x+(\beta(m)+n) y)}$ and expand the inside function, $m \mapsto e^{i 2 \pi \beta(m) y}$ according to the quasiperiodicity assumption on $\beta$ : specifically, 


$$
\begin{aligned}
e^{i 2 \pi \beta(m) y} & =\prod_{j=1}^{r} e^{i 2 \pi \xi_{j}(m) y} \\
& =\prod_{j=1}^{r} \sum_{k_{j} \in Z} c^{(j)}\left(k_{j}\right) \exp \left(i 2 \pi \frac{m k_{j}}{\omega_{j}}\right) \\
& =\sum_{k_{1} \in Z} \cdots \sum_{k_{r} \in \mathbb{Z}} c^{(1)}\left(k_{1}\right) \cdots c^{(r)}\left(k_{r}\right) \exp \left(i 2 \pi m \sum_{j=1}^{r} \frac{k_{j}}{\omega_{j}}\right) .
\end{aligned}
$$

Setting $c(k):=\prod_{j=1}^{r} c^{(j)}\left(k_{j}\right)$ and using

$$
\sum_{m \in Z} \exp \left[i 2 \pi m\left(x+\sum_{j=1}^{r} \frac{k_{j}}{\omega_{j}}\right)\right]=\sum_{m \in Z} \delta\left(x-\sum_{j=1}^{r} \frac{k_{j}}{\omega_{j}}-m\right)
$$

together with Poisson summation (also in the second variable) we arrive at the desired formula.

\section{HIGHER DIMENSIONS}

The following definitions help summarize the results for $d=2$ : We say that the one-parameter unitary groups on $\mathcal{L}^{2}(I \times I)$ generated by self-adjoint extensions of the respective partial derivatives $(1 / i)(\partial / \partial x)$ and $(1 / i)(\partial / \partial y)$ on $C_{c}^{\infty}(I \times I)$ are quasicommuting if the conditions (5.6) and (5.7) hold. Recall this means that the respective boundary operators commute with some phaseperiodic translation in the opposite variable. We then showed in Theorem V.1 that the commutativity property (5.3), for the unitary groups $U_{x}(s)$ and $U_{y}(t)$, implies quasicommutativity. Finally we showed in Corollary V.3 that, among the quasicommuting extensions, those that in fact commute [in the sense of (5.3)] are characterized by the two cocycle identities (5.8) and (5.9).

It is clear that quasicommutativity can be defined analogously for $d>2$. It follows from Theorem II.1 that commutativity of $d$ self-adjoint extensions of the respective partial derivatives $\left\{(1 / i)\left(\partial / \partial x_{j}\right): j=1, \ldots, d\right\}$, on $C_{c}^{\infty}\left(I^{d}\right) \subset \mathcal{L}^{2}\left(I^{d}\right)$, is equivalent to the spectral-pair condition for $\left(I^{d}, \Lambda\right)$. Moreover, if commuting self-adjoint extensions exist (i.e., $(1 / i)\left(\partial / \partial x_{j}\right) \subset H_{j}, H_{j}^{*}=H_{j}$, $j=1, \ldots, d)$, then we may take $\Lambda$ to be the joint spectrum of the family $\left\{H_{j}\right\}_{j=1}^{d}$. Conversely, commuting operators $H_{j}$ may easily be associated with some spectrum $\Lambda$ in a spectral pair $\left(I^{d}, \Lambda\right)$. Hence, for $d=2$, our results in Sec. V provide a complete classification of the commuting (and also the quasicommuting) self-adjoint extensions of $\left\{(1 / i)\left(\partial / \partial x_{j}\right)\right\}_{j=1}^{d}$.

In higher dimensions, we still have boundary operators corresponding to each self-adjoint extension of the partials $(1 / i)\left(\partial / \partial x_{j}\right)$ (on $\left.C_{c}^{\infty}\left(I^{d}\right) \subset \mathcal{L}^{2}\left(I^{d}\right), j=1, \ldots, d\right)$, by Corollary IV.2. If for each $j, U_{j}(t)$ denotes the unitary one-parameter group on $\mathcal{L}^{2}\left(I^{d}\right)$ generated by some self-adjoint extension $H_{j}$, then Corollary IV.2 states that $U_{j}(t)$ is induced by some unitary operator $V_{j}$ acting in the remaining variables $\left(x_{1}, \ldots, x_{j-1}, x_{j+1}, \ldots, x_{d}\right)$ (i.e., with omission of the variable on the $j$ th place): specifically, $U_{j}(t)=\operatorname{ind}_{\mathbb{Z}}^{\mathrm{R}}\left(V_{j}\right)$ as a representation of $(\mathbb{R},+)$; or equivalently the domain of $H_{j}$ is, for each $j$, given by the boundary condition

$$
f\left(x_{1} \ldots, x_{j-1}, 1, x_{j+1}, \ldots, x_{d}\right)=V_{j}\left(f\left(x_{1} \ldots, x_{j-1}, 0, x_{j+1}, \ldots, x_{d}\right)\right) .
$$

(Note that the more precise interpretation of this set of boundary conditions is given in formula (4.9) of Corollary IV.2. This is the interpretation of the unitary one-parameter groups in the respective coordinate directions as induced unitary representations (see Refs. 31 and 32), with induction $\mathbb{Z} \rightarrow \mathbb{R}$ for each direction.) We say that a family of self-adjoint extension operators $H_{j}$, with corresponding boundary unitaries $V_{j}$, is quasicommuting if there are phase angles $\alpha_{j}$ $\in[0,1\rangle, j=1, \ldots, d$, such that each $V_{j}$ is diagonalized by

$$
e_{\alpha_{1}+n_{1}}^{(1)} \otimes \cdots \otimes e_{\alpha_{j-1}+n_{j-1}}^{(j-1)} \otimes e_{\alpha_{j+1}+n_{j+1}}^{(j+1)} \otimes \cdots \otimes e_{\alpha_{d}+n_{d}}^{(d)}
$$


as $\left(n_{1}, \ldots, n_{d-1}, n_{d+1}, \ldots, n_{d}\right)$ vary over $\mathbb{Z}^{d-1}$, i.e., the lattice resulting from $\mathbb{Z}^{d}$ with the $j$ th coordinate place omitted. It follows that the quasicommutative case is characterized by the phase angles $\alpha_{1}, \ldots, \alpha_{d}$, and by functions $v_{j}: \mathbb{Z}^{d-1} \rightarrow \mathrm{T}$ such that, for $n=\left(n_{1}, \ldots, \hat{j}, \ldots, n_{d}\right), v_{j}(n)$ $=v_{j}\left(n_{1}, \ldots, \hat{j}, \ldots, n_{d}\right)$ is the eigenvalue of $V_{j}$ corresponding to the eigenvector in (7.1). (The notation $\left(n_{1}, \ldots, \hat{j}, \ldots, n_{d}\right)$ means that the $j$ th place is omitted.)

Theorem VII.1: Let $\left\{H_{j}\right\}_{j=1}^{d}$ be a family of self-adjoint extensions of the respective partials $(1 / i)\left(\partial / \partial x_{j}\right)\left(j=1, \ldots, d\right.$, on $\left.C_{c}^{\infty}\left(I^{d}\right) \subset \mathcal{L}^{2}\left(I^{d}\right)\right)$, which is assumed quasicommutative with eigenvalue functions $v_{j}\left(n_{1}, \ldots, \hat{j}, \ldots, n_{d}\right)$ from $\mathbb{Z}^{d-1}$ to $\mathbb{T}$. Then the extensions are commutative if and only if the following pair of cocycle conditions is satisfied for all $j, k$ such that $1 \leqslant j<k \leqslant d$, all $\left(n_{1}, \ldots, \hat{j}, \ldots, n_{d}\right)$, and all $l, m \in \mathbb{Z}\{0\}$ :

$$
\left(v_{j}\left(n_{1}, \ldots, \hat{j}, \ldots, n_{k}+l, \ldots, n_{d}\right)-v_{j}\left(n_{1}, \ldots, \hat{j}, \ldots, n_{d}\right)\right) \times\left(1-v_{k}\left(n_{1}, \ldots, \hat{k}, \ldots, n_{d}\right)\right)=0
$$

and

$$
\left(v_{k}\left(n_{1}, \ldots, n_{j}+m, \ldots, \hat{k}, \ldots, n_{d}\right)-v_{k}\left(n_{1}, \ldots, \hat{k}, \ldots, n_{d}\right)\right) \times\left(1-v_{j}\left(n_{1}, \ldots, \hat{j}, \ldots, n_{d}\right)\right)=0 .
$$

Proof: Since the commutativity for the one-parameter groups of unitary operators $U_{j}\left(t_{j}\right)$ may be stated for pairs, i.e., $U_{j}\left(t_{j}\right) U_{k}\left(t_{k}\right)=U_{k}\left(t_{k}\right) U_{j}\left(t_{j}\right), j<k, t_{j} \in \mathbb{R}, t_{k} \in \mathbb{R}$, the argument for the general case $d>2$ is the same as for $d=3$. To see this, just use the formulas for the respective one-parameter groups which are analogues to (5.12) and (5.13) in the proof of Corollary V.4. For $d=3$, we may introduce the leg-notation: $v_{1} \rightarrow v_{23}, v_{2} \rightarrow v_{13}, v_{3} \rightarrow v_{12}$. When evaluated at a general point in $\mathbb{Z}^{3}$ of the form $(k, l, m)$, the respective eigenvalues are

$$
\begin{aligned}
& v_{23}(l, m) \text { for } V_{23}, \\
& v_{13}(k, m) \text { for } V_{13}, \\
& v_{12}(k, l) \text { for } V_{12} .
\end{aligned}
$$

Specifically,

$$
\begin{gathered}
V_{23} e_{\beta+l}^{(2)} \otimes e_{\gamma+m}^{(3)}=v_{23}(l, m) e_{\beta+l}^{(2)} \otimes e_{\gamma+m}^{(3)}, \\
V_{13} e_{\alpha+k}^{(1)} \otimes e_{\gamma+m}^{(3)}=v_{13}(k, m) e_{\alpha+k}^{(1)} \otimes e_{\gamma+m}^{(3)}, \\
V_{12} e_{\alpha+k}^{(1)} \otimes e_{\beta+l}^{(2)}=v_{12}(k, l) e_{\alpha+k}^{(1)} \otimes e_{\beta+l}^{(2)},
\end{gathered}
$$

where $\alpha, \beta, \gamma$ are the fixed phase angles from the quasicommutativity. Then the three pairs of cocycle identities from the theorem are as follows: (ia)-(ib), (iia)-(iib), and (iiia)-(iiib). The argument for the equivalence of commutativity and the cocycle identities is essentially the same as the one used in the proof of Corollary V.4 above. The cocycle identities for $d=3$ are

$$
\begin{gathered}
\left(v_{13}(k, m)-v_{13}\left(k+n_{1}, m\right)\right)\left(1-v_{23}(l, m)\right)=0, \\
\left(v_{23}(l, m)-v_{23}\left(l+n_{2}, m\right)\right)\left(1-v_{13}(k, m)\right)=0, \\
\left(v_{12}(k, l)-v_{12}\left(k+n_{1}, l\right)\right)\left(1-v_{23}(l, m)\right)=0, \\
\left(v_{23}(l, m)-v_{23}\left(l, m+n_{3}\right)\right)\left(1-v_{12}(k, l)\right)=0,
\end{gathered}
$$

and

$$
\left(v_{13}(k, m)-v_{13}\left(k, m+n_{3}\right)\right)\left(1-v_{12}(k, l)\right)=0,
$$




$$
\left(v_{12}(k, l)-v_{12}\left(k, l+n_{2}\right)\right)\left(1-v_{13}(k, m)\right)=0 .
$$

Example VII.2: Not all the spectral pairs in three dimensions are quasicommutative (although this is true in $d=2$ ). Take for example the case (3.4) of Sec. III with

$$
\Lambda=\left\{\left(\begin{array}{c}
k \\
\beta(k)+l \\
\gamma(k, l)+m
\end{array}\right): k, l, m \in \mathbb{Z}\right\}
$$

with $\beta: \mathbb{Z} \rightarrow[0,1\rangle$ and $\gamma: \mathbb{Z}^{2} \rightarrow[0,1\rangle$ arbitrarily given functions. Then the three operators $V_{23}, V_{13}$, and $V_{12}$ are as follows:

$V_{23}=I$ (the identity operator in the two marked tensor slots),

$$
V_{13}\left(e_{k}^{(1)} \otimes e_{\gamma(k, l)+m}^{(3)}\right)=e^{i 2 \pi \beta(k)} e_{k}^{(1)} \otimes e_{\gamma(k, l)+m}^{(3)},
$$

and

$$
V_{12}\left(e_{k}^{(1)} \otimes e_{\beta(k)+l}^{(2)}\right)=e^{i 2 \pi \gamma(k, l)} e_{k}^{(1)} \otimes e_{\beta(k)+l}^{(2)}
$$

It follows that the three commuting unitary one-parameter groups associated with $\Lambda$, via Theorem II.1, are not quasicommuting if the two functions $\beta$ and $\gamma$ in formula (7.4) are both nonconstant.

\section{ACKNOWLEDGMENTS}

Work supported by the National Science Foundation. The authors gratefully acknowledge excellent typesetting and graphics production by Brian Treadway.

${ }^{1}$ M. S. Livšic, N. Kravitsky, A. S. Markus, and V. Vinnikov, Theory of Commuting Non-selfadjoint Operators, Mathematics and its Applications Vol. 332 (Kluwer Academic, Dordrecht, 1995).

${ }^{2}$ S. A. Avdonin and S. A. Ivanov, Families of Exponentials: The Method of Moments in Controllability Problems for Distributed Parameter Systems (Cambridge University Press, Cambridge, 1995).

${ }^{3}$ M. I. Belishev, "Wave bases in multidimensional inverse problems," Mat. Sb. 180, 584-602 (1989); 180, 720 (1989); (Russian), English translation: Math. USSR-Sb. 67, 23-42 (1990).

${ }^{4}$ A. Haraux and V. Komornik, "On the vibrations of rectangular plates," Proc. R. Soc. Edinburgh, Sect. A: Math. 119, 47-62 (1991).

${ }^{5}$ B. S. Pavlov, "The basis property of a system of exponentials and the condition of Muckenhoupt," Dokl. Akad. Nauk SSSR 247, 37-40 (1979), English translation: Soviet Math. Dokl. 20, 655-659 (1979).

${ }^{6}$ R. T. Powers, "Self-adjoint algebras of unbounded operators," Commun. Math. Phys. 21, 85-124 (1971).

${ }^{7}$ R. T. Powers, "Selfadjoint algebras of unbounded operators, II," Trans. Am. Math. Soc. 187, 261-293 (1974).

${ }^{8}$ A. Kempf, "A generalized Shannon sampling theorem, fields at the Planck scale as bandlimited signals" (unpublished); hep-th/9905114.

${ }^{9}$ A. Kempf, "Unsharp degrees of freedom and the generating of symmetries"' (unpublished), hep-th/9907160.

${ }^{10} \mathrm{P}$. E. T. Jorgensen and S. Pedersen, "Spectral theory for Borel sets in $\mathrm{R}^{n}$ of finite measure," J. Funct. Anal. 107, 72-104 (1992).

${ }^{11}$ P. E. T. Jorgensen and S. Pedersen, "Harmonic analysis and fractal limit-measures induced by representations of a certain $C^{*}$-algebra," J. Funct. Anal. 125, 90-110 (1994).

${ }^{12}$ P. E. T. Jorgensen and S. Pedersen, "Harmonic analysis of fractal measures," Constr. Approx. 12, 1-30 (1996).

${ }^{13}$ B. Fuglede, "Commuting self-adjoint partial differential operators and a group theoretic problem," J. Funct. Anal. 16, 101-121 (1974).

${ }^{14}$ P. E. T. Jorgensen, "Spectral theory of finite volume domains in $\mathbb{R}^{n}$," Adv. Math. 44, 105-120 (1982).

${ }^{15}$ S. Pedersen, "Spectral theory of commuting self-adjoint partial differential operators," J. Funct. Anal. 73, 122-134 (1987).

${ }^{16}$ M. Reed and B. Simon, Fourier Analysis, Selfadjointness, Methods of Modern Mathematical Physics Vol. II (Academic, New York, 1975).

${ }^{17}$ N. Dunford and J. T. Schwartz, Linear Operators (Wiley Interscience, New York, 1963), Vol. II.

${ }^{18}$ J. Friedrich, "On first order partial differential operators on bounded regions of the plane," Math. Nachr. 131, 33-47 (1987). 
${ }^{19}$ J. von Neumann, “Allgemeine eigenwerttheorie Hermitescher funktionaloperatoren," Math. Ann. 102, 49-131 (1929/ $30)$.

${ }^{20}$ J. C. Lagarias and Y. Wang, "Tiling the line with translates of one tile,"' Invent. Math. 124, 341-365 (1996).

${ }^{21}$ J. C. Lagarias and Y. Wang, "Integral self-affine tiles in R ${ }^{n}$. II. Lattice tilings," J. Fourier Anal. Appl. 3, 83-102 (1997).

${ }^{22}$ Ergodic Theory, Symbolic Dynamics, and Hyperbolic Spaces (Trieste, 1989), edited by T. Bedford, M. Keane, and C. Series (Oxford University Press, New York, 1991).

${ }^{23}$ P. E. T. Jorgensen and S. Pedersen, "Spectral pairs in Cartesian coordinates," J. Fourier Analysis Applications 5, 285-302 (1999).

${ }^{24}$ P. E. T. Jorgensen, "Selfadjoint extension operators commuting with an algebra," Math. Z. 169, 41-62 (1979).

${ }^{25}$ P. E. T. Jorgensen, "Commutative algebras of unbounded operators," J. Math. Anal. Appl. 123, 508-527 (1987).

${ }^{26}$ P. E. T. Jorgensen, "Extensions and index of Hermitian representations," Publ. Res. Inst. Math. Sci. 25, 923-945 (1989).

${ }^{27}$ S. Pedersen, "Spectral sets whose spectrum is a lattice with a base," J. Funct. Anal. 141, 496-509 (1996).

${ }^{28}$ M. Senechal, Quasicrystals and Geometry (Cambridge University Press, Cambridge, 1995).

${ }^{29}$ A. Hof, "On diffraction by aperiodic structures," Commun. Math. Phys. 169, 25-43 (1995).

${ }^{30}$ E. Bombieri and J. E. Taylor, "Quasicrystals, tilings, and algebraic number theory: Some preliminary connections," pp. 241-264 in The Legacy of Sonya Kovalevskaya (Cambridge, MA and Amherst, MA, 1985), Contemporary Mathematics Vol. 64, edited by L. Keen (American Mathematical Society, Providence, 1987).

${ }^{31}$ G. W. Mackey, "Induced representations of locally compact groups. II. The Frobenius reciprocity theorem,'” Ann. Math. 58, 193-221 (1953).

${ }^{32}$ G. W. Mackey, "Point realizations of transformation groups,” Ill. J. Math. 6, 327-335 (1962). 